\title{
ROLE OF OPTICAL COHERENCE TOMOGRAPHY IN CENTRAL SEROUS CHORIORETINOPATHY
}

Junaid Salam Wani ${ }^{1}$, Parvez Ahmad Bhat ${ }^{2}$, Andleeb Ahangar 3 , Suraya Ismail ${ }^{4}$

\section{HOW TO CITE THIS ARTICLE:}

Junaid Salam Wani, Parvez Ahmad Bhat, Andleeb Ahangar, Suraya Ismail. "Role of Optical Coherence Tomography in Central Serous Chorioretinopathy". Journal of Evolution of Medical and Dental Sciences 2015; Vol. 4, Issue 45, June 04; Page: 7801-7809, DOI: 10.14260/jemds/2015/1135

ABSTRACT: PURPOSE: To study the role of optical coherence tomography in central serous chorioretinopathy. MATERIAL AND METHODS: A total of 48 eyes of 48 patients with a clinical diagnosis of central serous chorioretinopathy were evaluated with spectral domain optical coherence tomography. After detailed ocular examination, followed by fundus fluorescein angiography, optical coherence tomography was performed and various anatomic features were noted. RESULTS: Out of 48 eyes, 41 had acute and 7 had chronic central serous chorioretinopathy. The mean age of the patients was 35 (SD7.1) years with male (83.4\%) preponderance in our study. All patients had unilateral involvement with blurring of vision (43.75\%) and metamorphopsia (31.25\%) being the main presenting complaints. Fundus fluorescein angiography revealed a total of 56 leakage points with ink- blot pattern being 6 times more frequent than smoke-stack pattern in our study. On optical coherence tomography neurosensory detachment was seen in 44 eyes. Total macular thickness ranged from $220 \mu \mathrm{m}-1120 \mu \mathrm{m}$ (mean \pm SD:447 \pm 219 ) and vault height ranged from $100 \mu \mathrm{m}-900 \mu \mathrm{m}$ (mean \pm SD:350 187 ). Retinal pigment epithelial abnormality was seen in $87.5 \%$ eyes, retinal pigment epithelial detachment in $18.75 \%$ eyes and retinal pigment epithelial bulge in $27.09 \%$ patients. CONCLUSION: Optical coherence tomography can be used as a complimentary diagnostic tool in central serous chorioretinopathy.

KEYWORDS: Optical Coherence Tomography, Central Serous Chrorioretinopathy, Fundus Fluorescein Angiography.

INTRODUCTION: Central serous chorioretinopathy is a chorioretinal disorder, incompletely understood, with systemic associations, multifactorial etiology and complex pathogenesis. Characterised by serous neurosensory detachment usually located at the posterior pole of the retina, this disorder typically affects young to middle aged men with type A personality. Although an idiopathic condition associated with stress it might also be secondary to high exogenous and endogenous corticosteroids.[1] Central serous chorioretinopathy is a self-limiting disorder, with spontaneous resolution and good prognosis. However some cases may suffer from persistent or recurrent serous macular detachment with subsequent progressive visual loss.

Patients with central serous chorioretinopathy present with diminution of vision, metamorphopsia, relative central scotoma, micropsia, dyschromatopsia and blurring of vision. Secondary to the anterior displacement of the detached neurosensory retina, the eye becomes hyperopic and vision is often improved with use of convex lens. Fundus examination reveals a well demarcated area of neurosensory retinal detachment at the posterior pole which is confirmed by slit lamp biomicroscopy. Yellow dots are frequently observed on the posterior surface of the detached retina and are secondary to phagocytosis and shed photoreceptor outer segments.[2] Yellowish discolouration of the fovea may be seen which is due to increased visibility of the retinal xanthophyll. 


\section{ORIGINAL ARTICLE}

For years fluorescein angiography has been the main confirmatory investigation for central serous chorioretinopathy with typical presence of one or more leakage points in the retinal pigment epithelium, namely ink- blot and smoke-stack pattern of dye leak. Chronic central serous chorioretinopathy might show a retinal pigment epithelium window defect due to retinal pigment epithelium atrophy, as they usually lack the typical leakage pattern as seen in adults. Fundus fluorescein angiography is helpful in understanding the physiological aspect of this disorder.

However the availability of optical coherence tomography has vastly enhanced the anatomical assessment and understanding of central serous chorioretinopathy by providing cross sectional imaging of the macula. It is a non-contact, non-invasive imaging system which uses reflection of light off retinal layers to create false colour tomographic images of the retinal and retinal pigment epithelium structures enabling to assess the anatomical pattern of the disease. Optical coherence tomography demonstrates presence of sub retinal fluid or pigment epithelial defect and helps in differentiation between the two.[3] optical coherence tomography is a useful in detecting anatomical changes which are not evident on clinical examination and serial scans can be taken to assess disease progression and response to treatment.

In this study we evaluated patients with clinical diagnosis of central serous chorioretinopathy using optical coherence tomography and tried to describe characteristic features observed during this study.

MATERIALS AND METHODS: In this study a total of 48 eyesof48 patients with a clinical diagnosis of central serous chorioretinopathy, were evaluated with spectral domain optical coherence tomography between June 2011 to June 2013 in the department of ophthalmology. Ethics committee approval was sought and informed consent was obtained from all patients.

Inclusion criteria of the patients were a history of sudden central vision loss, metamorphopsia, micropsia, characteristic ring reflex on indirect ophthalmoscopy and a characteristic leakage pattern on fundus fluorescein angiography. Exclusion criteria were any other macular abnormality like choroidal neovascularisation, age related macular degeneration or any other macular degeneration.

A detailed history was taken. Ocular examination included visual acuity testing using snellen's chart, slit lamp examination, intraocular pressure measurement using applanation tonometry, amsler grid examination, cycloplegic refraction and slit lamp biomicroscopy. Fundus fluorescein angiography was done on the same day of presentation.

The patients with clinical diagnosis of central serous chorioretinopathy and characteristic leakage pattern on fundus fluorescein angiography were evaluated using spectral domain optical coherence tomography. Optical coherence tomography findings with regard to neurosensory detachment, pigment epithelial defects (Pigment epithelial defect) or bulge, hyper-reflective deposits, were recorded and analyzed. Total macular thickness was recorded as the distance between superior border of retinal pigment epithelium and superior border of detached neurosensory retina at the Centre of macula. Vault height was measured as the distance between the superior border of retinal pigment epithelium and inferior border of the detached neurosensory retina and corresponds to the height of sub retinal fluid at the center of macula.

RESULTS: In the present study, 48 eyes of 48 patients of central serous chorioretinopathy were evaluated. 41 patients presented with acute central serous chorioretinopathy while 7 patients had 


\section{ORIGINAL ARTICLE}

chronic central serous chorioretinopathy. The mean age of the patients was 35(SD 7.1) years, with a range 26 to 50 years. Out of 48 patients there were $40(83.4 \%)$ males and $8(16.66 \%)$ females (Table 1). All the 48 patients had unilateral involvement of eye with 23(47.91\%) patients presenting with central serous chorioretinopathy in the right eye and 25(52.08\%) had central serous chorioretinopathy in left eye (Table 2). Out of 48 patients, $41(85.41 \%$ ) had acute central serous chorioretinopathy and $7(14.54 \%)$ had chronic central serous chorioretinopathy. Blurring of vision and metamorphopsia were the main presenting complaints followed by relative scotoma and micropsia. 21(43.75\%) patients presented with blurring of vision whereas $15(31.25 \%)$ presented with metamorphopsia. $9(18.75 \%)$ patients presented with relative scotoma and only $3(6.25 \%)$ patients had chief complaint of micropsia (Table 2). The visual acuity of most of the patients $(93.75 \%)$ was in the range of $6 / 6 \mathrm{P}$ to $6 / 36.6 .25 \%$ patients had avisual acuity of $6 / 60$ or less (Table 3).

On fundus fluorescein angiography 48 eyes of 48 patients with central serous chorioretinopathy revealed 56 leakage points. The leakage patterns observed were either ink- blot or smoke-stack type, the former being 6 times more frequent. Out of 48 eyes, 43 eyes showed single leakage point, 4 eyes showed double and one eye showed multiple leakage points (Table 4). Most of the leakages occurred in superonasal quadrant in 22(39.28\%) patients followed by inferonasal in $14(25 \%)$ patients and inferotemporal in $6(10.71 \%)$ patients. Leakage inferior to macula was seen only in $2(3.57 \%)$ patients.

Optical coherence tomography showed neurosensory detachment in all 41 eyes with acute central serous chorioretinopathy and only in 3 out of 7 eyes with chronic central serous chorioretinopathy. Total macular thickness ranged from $220 \mu \mathrm{m}$ to $1120 \mu \mathrm{m}$ (mean \pm SD:447 \pm 219 ). Vault height was measured in only 44 eyes because only 44 eyes showed neurosensory detachment in our study. It ranged from $100 \mu \mathrm{m}$ to $940 \mu \mathrm{m}$ (mean \pm SD:350 $\mu \mathrm{m} \pm 187 \mu \mathrm{m}$ ). Out of 48 eyes of central serous chorioretinopathy, 42(87.5\%) eyes showed some kind of retinal pigment epithelium abnormality on optical coherence tomography. retinal pigment epithelium detachment (Pigment epithelial defect) was seen in $9(18.75 \%)$ patients and retinal pigment epithelium bulge in $13(27.09 \%)$ patients. $20(41.66 \%)$ patients had irregular retinal pigment epithelium. $6(14.63 \%)$ eyes showed hyper reflective subretinal deposits on optical coherence tomography. These deposits were more intense and numerous in chronic central serous chorioretinopathy.

DISCUSSION: Traditionally central serous chorioretinopathy patients were diagnosed by slit lamp biomicroscopy and by fundus fluorescein angiography. The introduction of optical coherence tomography gave one the ability to non-invasively diagnose and follow patients over time.

Majority of the patients in the present study were male (83.4\%), the ratio between males and females being 5:1 (Table 1). The youngest and the oldest patient in the study were 26 and 50 year old respectively (Range $26-50$ years with mean \pm SD:35 \pm 7.1 ). The highest number of patients was seen in the age group 26 to 35 years and least number was seen in 40 to 50 years of age group. It shows that this disease has a predilection for younger age (Table 1). Georage Benette, ${ }^{[4]}$ in his study of 27 cases of central serous chorioretinopathy, found preponderance of males compared to females with a ratio of 4.5:1, which is approximately similar to our observations. He also found maximum number of cases were in the age group of 25 to 44 years.

All the patients in our study had unilateral presentation. The right eye and the left eye were involved approximately with the same frequency (Table 2). In our study the commonest presenting 


\section{ORIGINAL ARTICLE}

complaint was blurring of vision (43.75\%), followed by metamorphopsia (31.25\%), relative scotoma (18.75\%) and micropsia (6.25\%) (Table2). Yamda,[5] et al studied 53 patients of central serous chorioretinopathy and the commonest presenting complaint in their study was relative scotoma (58\%), followed by blurring of vision (34\%), metamorphopsia (18\%), micropsia (13.2\%) and xanthopsia (5.7\%), which is approximately similar to our study except that we found blurring of vision as the most common complaint and no patient in our study complained of xanthopsia. In our study out of 48 patients 7 had chronic central serous chorioretinopathy (duration more than 3 months) and 41 patients had acute central serous chorioretinopathy (Duration less than 2 weeks). 2 patients out of 48 patients had a history of similar episode in the fellow eye in past, which had recovered fully without any residual defect. Visual acuity of the patients was measured on Snellen's chart ranged from 6/6P to 6/60. No patient had visual acuity less than 6/60. Majority of the patients (85.41\%) had a visual acuity between 6/9-6/36 (Table 3).

Fundus fluorescein angiography was done in all 48 patients and revealed a total of 56 leakage points, which were seen as hyperfluorescent spots. These hyperfluorescent spots assumed a characteristic shape and were accordingly classified as having either an inkblot or smoke stack type of leak. Out of 56 leakage points in 48 eyes, 48(85.7\%) were inkblot (Fig. 1a) and 8(14.28\%) were smoke stack (Fig. 1b) type of leak (Table-4). Yamda, ${ }^{[5]}$ et al reported in their study, 62.2\% inkblot type of leakage which is lower than our figure of $85.7 \%$ and $24.62 \%$ of smoke stack type which is higher than our figure of $14.28 \%$. Majority of the eyes in our study (89.58\%) showed a single leakage point, $8.33 \%$ eyes showed double leakage point and only one eye $(1.785 \%)$ showed multiple leakage points. So it was seen that inkblot type of leakage was six times more frequent than smoke stack type.

Optical coherence tomography is a high resolution imaging technique that provides cross sectional tomographic images of the ocular microstructures through the thickness of retina. It is analogous to ultrasound measuring the back reflection intensity of infra-red light rather than sound. An optical coherence tomography image is a two dimensional data set that represents difference in optical visualization. Optical coherence tomography images can be analyzed qualitatively and quantitatively to detect retinal abnormalities. As a result of providing detailed information on the architectural morphology of retina at the level of individual retinal layers, optical coherence tomography has been proposed to detect early pathological changes even before clinical symptoms and visual signs occur. In our study we performed optical coherence tomography of 48 cases of central serous chorioretinopathy and documented the following observations.

Majority (91.66\%) of the patients had neurosensory detachment (Fig. 2a) on optical coherence tomography. These included all the 41 eyes with acute central serous chorioretinopathy and 3 eyes with chronic central serous chorioretinopathy. Remaining 4 eyes with chronic central serous chorioretinopathy had no neurosensory detachment but showed retinal pigment epithelium abnormality with sub-retinal hyper-reflective deposits. Total macular thickness was measured in all the 48 eyes and was in the range of $220 \mu \mathrm{m}-1120 \mu \mathrm{m}$ (Mean \pm SD:447 \pm 219 ). Vault height corresponding to the height of the fluid under the sensory retina was measured in only 44 eyes with neurosensory detachment on optical coherence tomography.

It was found in the range of $100 \mu \mathrm{m}$ to $940 \mu \mathrm{m}$ (Mean \pm SD:350 \pm 18.7 ) (Table-5). $87.5 \%$ patients showed some kind of retinal pigment epithelium abnormality on optical coherence tomography and included retinal pigment epithelium detachment in $18.75 \%$, retinal pigment epithelium Bulge (Fig. 2b) in $27.09 \%$ and Irregular retinal pigment epithelium in $41.60 \%$. These changes were seen in both acute and chronic cases. 


\section{ORIGINAL ARTICLE}

It was observed that optical coherence tomography is especially useful in detecting shallow sub retinal fluid and small pigment epithelial defect, which might be difficult to identify clinically. With the help of optical coherence tomography legions like retinal pigment epithelium bumps, bulges and small pigment epithelial defects can be demonstrated.[6]

The concept of diffuse retinal pigment epithelium involvement is supported by number of sources. Two recently published articles confirm the observation of multiple pigment epithelial defects and other retinal pigment epithelium abnormalities on optical coherence tomography in patients with central serous chorioretinopathy.[7] Out of 42 eyes that retinal pigment epithelium abnormality on optical coherence tomography, in 31(73.5\%) eyes this retinal pigment epithelium abnormality exactly coincided with leakage points on fundus fluorescein angiography, which shows that it is this retinal pigment epithelium defect through which fluid leaks from choroid into the sub retinal space. Perkin,[8] et al postulated that pigment epithelial defects in central serous chorioretinopathy are a sign that larger areas of compromised retinal pigment epithelium are involved. Focal leaks or defects in the retinal pigment epithelium with normal surrounding epithelial cells do not give rise to sub retinal fluid leakage.

In presence of dysfunctional retinal pigment epithelium there is reduced active transport of fluid from retina towards choroid allowing accumulation of sub retinal fluid; another argument for more widespread retinal pigment epithelium dysfunction. We also observed hyper reflective deposits located deep to neurosensory retina in all $7(100 \%)$ eyes with chronic central serous chorioretinopathy (Fig. 2c) and 6(14.63\%) eyes with acute central serous chorioretinopathy, however they were more intense in chronic cases. These may represent lumping of proteins as their concentration increases in the resolving fluid. ${ }^{[9]}$ Another explanation might be that these are cellular segments, but highly reflective nature of these spots on optical coherence tomography scans make this less likely. Perkin, ${ }^{[8]}$ et al found a strong association between final recovery of visual acuity and the presence of sub retinal deposits on optical coherence tomography. They found that in patients with more intense sub retinal deposits, there was some deficit in the final visual acuity, once the sub retinal fluid resolved.

Unlike fundus fluorescein angiography, optical coherence tomography being a non-invasive, non-contact, safe diagnostic tool with no contraindications, can be repeated and serial scans can be used to assess progression of disease and treatment response. Quantitative measurement and thickness maps are useful for documentation as well as for research purpose. Optical coherence tomography can also offer valuable prognostic information, as it can demonstrate cystoid degeneration, disruption of the outer photoreceptors and inner/outer segment junction, which are associated with poor visual outcome.[10] Hence optical coherence tomography can be used as a complimentary diagnostic tool for evaluation of patients of central serous chorioretinopathy and can be used as a primary diagnostic procedure for patients in whom fundus fluorescein angiography is contraindicated

CONCLUSION: Optical coherence tomography is a safe diagnostic tool with no side effects and can be used as a complimentary investigative procedure to diagnose, access progression and response to treatment in case of central serous retinopathy. It can also be used as a primary diagnostic procedure when central serous chorioretinopathy is suspected in pregnant women and patients in whom fundus fluorescein angiography is contraindicated. 
ORIGINAL ARTICLE

\begin{tabular}{|c|c|c|c|}
\hline & & $\mathbf{n}$ & $\mathbf{\%}$ \\
\hline Age (Years) & $26-30$ & 15 & 31.25 \\
\hline & $31-35$ & 19 & 39.58 \\
\hline & $36-40$ & 9 & 18.75 \\
\hline & $41-45$ & 4 & 8.33 \\
\hline & $46-50$ & 1 & 2.08 \\
\hline & $>50$ & 0 & 0.00 \\
\hline Mean \pm SD & $35 \pm 7.1$ & & \\
\hline & & & \\
\hline Gender & Male & 40 & 83.34 \\
\hline & Female & 8 & 16.66 \\
\hline
\end{tabular}

Table 1: Age and sex Distribution

\begin{tabular}{|c|c|c|c|}
\hline & & n & \% \\
\hline Laterality & Unilateral & 48 & 100.00 \\
\hline & Bilateral & 0 & 0.00 \\
\hline & Right eye & 23 & 47.91 \\
\hline & Left eye & 25 & 52.08 \\
\hline & & & \\
\hline Presenting Complaint & Blurring of vision & 21 & 43.75 \\
\hline & Metamorphopsia & 15 & 31.25 \\
\hline & Relative scotoma & 9 & 18.75 \\
\hline & Micropsia & 3 & 6.25 \\
\hline
\end{tabular}

Table 2: Clinical Features

\begin{tabular}{|c|c|c|}
\hline Visual Acuity & $\mathbf{n}$ & $\mathbf{\%}$ \\
\hline $6 / 6 \mathrm{P}$ & 4 & 8.33 \\
\hline $6 / 9-6 / 9 \mathrm{P}$ & 9 & 18.75 \\
\hline $6 / 12-6 / 12 \mathrm{P}$ & 13 & 27.08 \\
\hline $6 / 18-6 / 18 \mathrm{P}$ & 12 & 25.00 \\
\hline $6 / 24-6 / 24 \mathrm{P}$ & 0 & 0.00 \\
\hline $6 / 36-6 / 36 \mathrm{P}$ & 7 & 14.58 \\
\hline $6 / 60$ & 3 & 6.25 \\
\hline$<6 / 60$ & 0 & 0.00 \\
\hline
\end{tabular}

Table 3: Visual Acuity on Presentation

\begin{tabular}{|c|c|c|}
\hline No. of leakage points & Ink-blot Pattern & Smoke-stack Pattern \\
\hline Single & 38 & 5 \\
\hline Double & 1 & 3 \\
\hline More than 2 & 1 & 0 \\
\hline Total leakage points & $\mathbf{4 0}(\mathbf{8 7 . 7 1 \% )}$ & $\mathbf{8 ( 1 4 . 2 8 \% )}$ \\
\hline \multicolumn{2}{|r}{ Table 4: Fundus Fluorescein Angiography } \\
\hline
\end{tabular}


ORIGINAL ARTICLE

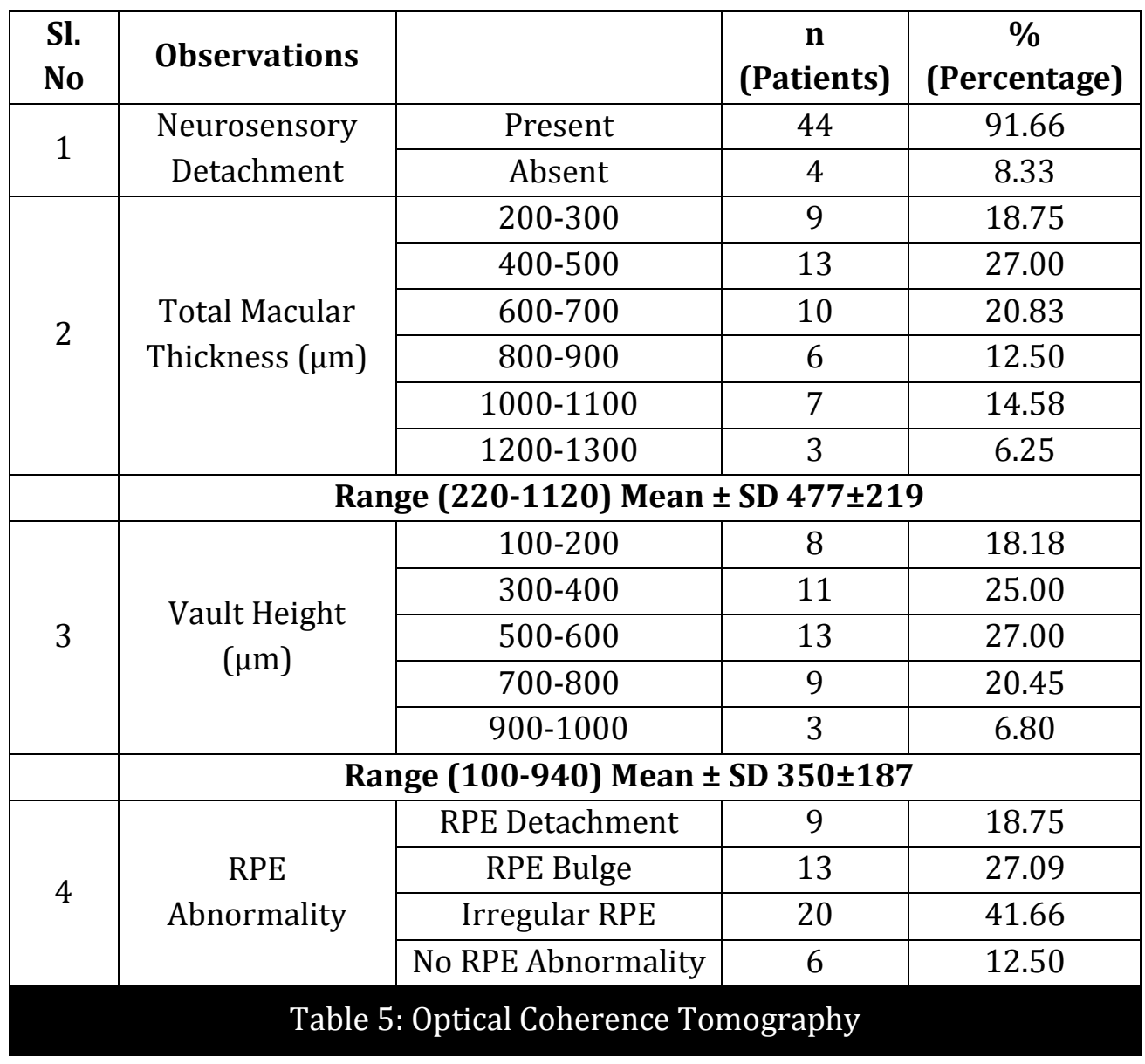

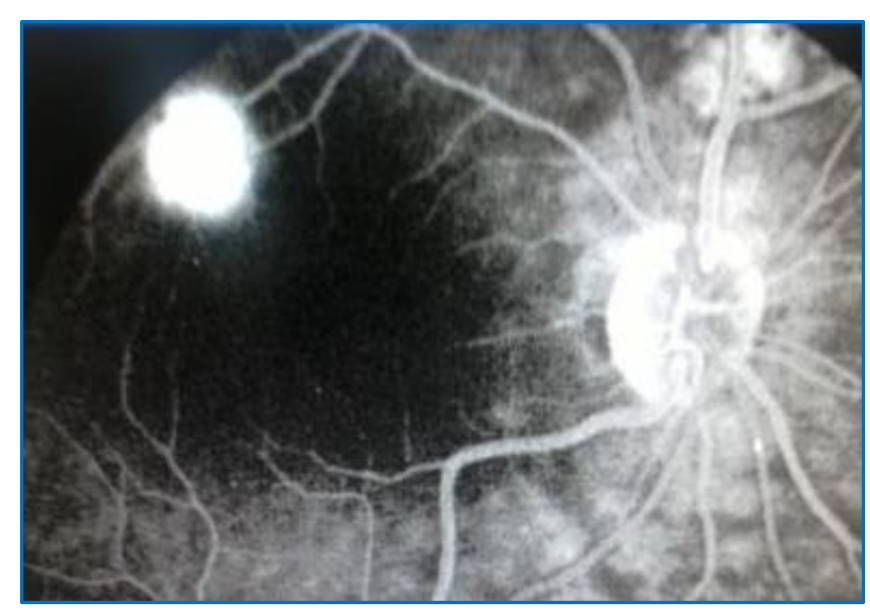

Fig. 1 a

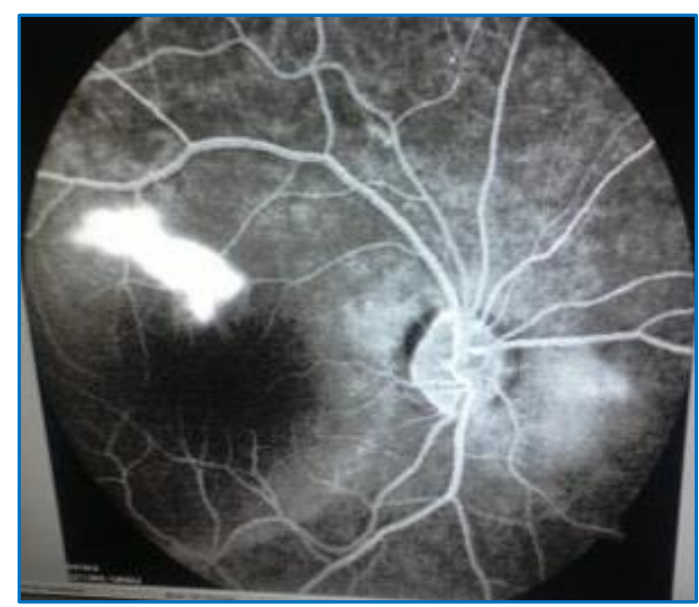

Fig. 1 b 


\section{ORIGINAL ARTICLE}

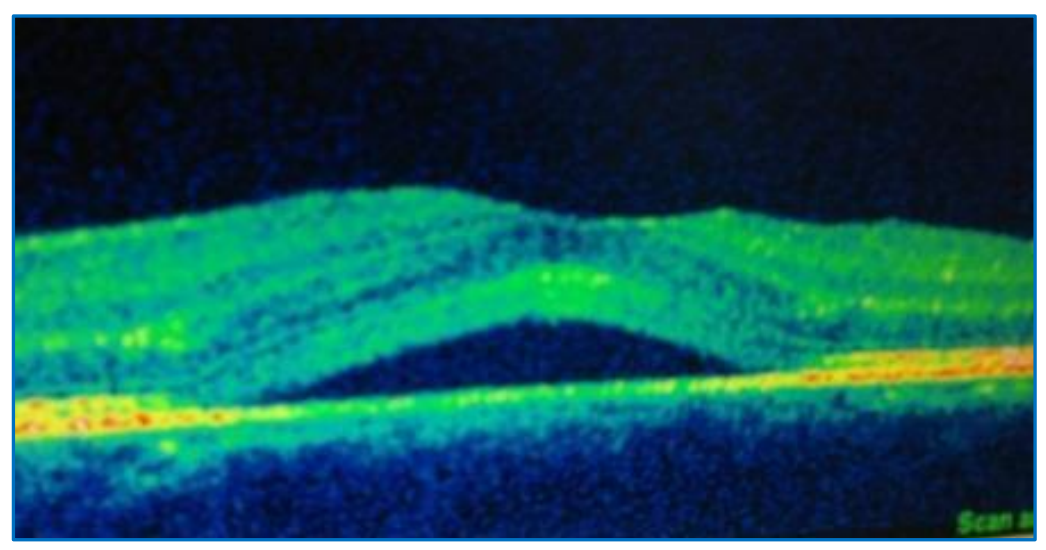

Fig. 2a

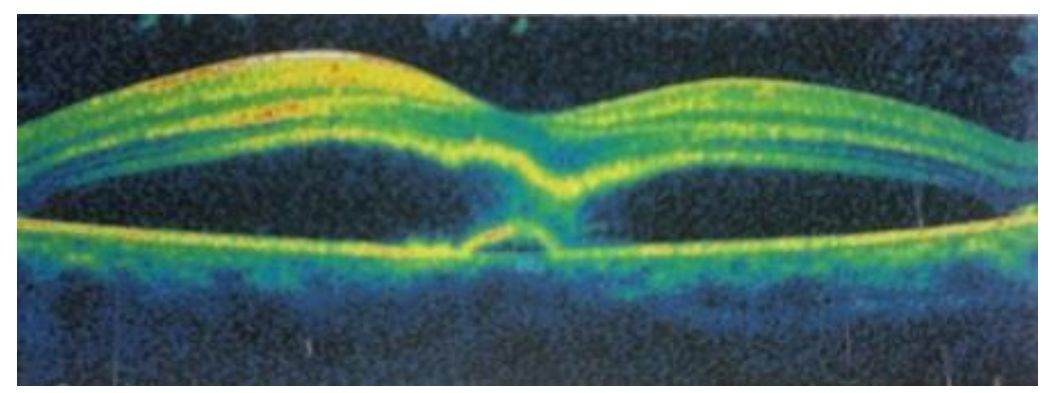

Fig. 2 b

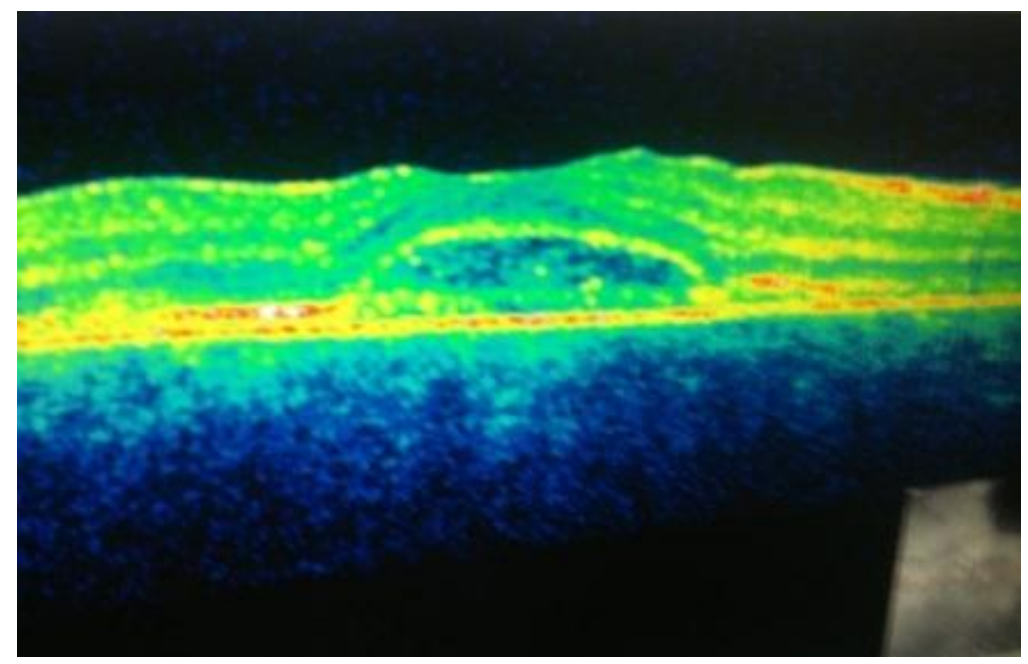

Fig. 2c 


\section{BIBLIOGRAPHY:}

1. Zakir SM, Shukla M, Simi ZU, Ahmad J, Sajid M. Serum cortisol and testosterone levels in idiopathic central serous chorioretinopathy. Indian J Ophthalmol 2009 0ct; 57(6): 419-22.

2. Maruko I, Iida T, Ojima A, Sekiryu T. Subretinal dot-like precipitates and yellow material in central serous chorioretinopathy. Retina 2011 Apr; 31(4): 759-65.

3. Hee MR, Puliafito CA, Wong C, Reichel E, Duker JS, Schuman JS et al. Optical coherence tomography of central serousretinopathy. Am J Ophthalmol 1995 Jul; 120(1): 65-74.

4. Benett Georage. Central Serous Retinopathy. Br J Ophthalmol 1955 Oct; 39 (10): 605-18.

5. Yamda K, Hayasaka S, Setogowa T. Fluorescein-angiographic patterns in patients with central serous chorioretinopathy at initial visit. Ophthalmologica 1992; 205(2): 69-76.

6. Gupta P, Gupta V, Dogra MR, Singh R, Gupt A. Morphological changes in the retinal pigment epithelium on spectral- domain OCT in the unaffected eyes with idiopathic central serous chorioretinopathy. Int Ophthalmol 2010 Apr; 30(2): 175-81.

7. Sawa M, Gomi F, Harino S, Ohguro N, Ohji M, Tano Y. Three-Dimensional optical coherence tomographic findings of idiopathic multiple serous retinal pigment epithelial detachments. Arch Ophthalmol 2005 Jan; 123(1): 122-3.

8. Perkin SL, Kim JE, Pollack JS, Merrill PT. Clinical characteristics of central serous chorioretinopathy in women. Ophthalmology 2002 Feb; 109(2): 262-6.

9. Gass JDM. Pathogenesis of disciform detachment of the neuroepitheium. II. Idiopathic central serous choroidopathy. Am J Ophthalmol 1967; 63: 587-15.

10. Kim YY, Flaxel CJ. Factors influencing the visual acuity of chronic central serous chorioretinopathy. Korean J Ophthalmol 2011 Apr; 25 (2): 90-7.

\section{AUTHORS:}

1. Junaid Salam Wani

2. Parvez Ahmad Bhat

3. Andleeb Ahangar

4. Suraya Ismail

\section{PARTICULARS OF CONTRIBUTORS:}

1. Associate Professor, Department of Ophthalmology, Government Medical College, Srinagar.

2. Resident, Department of Ophthalmology, Government Medical College, Srinagar.

3. Resident, Department of Ophthalmology, Government Medical College, Srinagar.

FINANCIAL OR OTHER COMPETING INTERESTS: None
4. Resident, Department of Opthalmology, Government Medical College, Srinagar.

\section{NAME ADDRESS EMAIL ID OF THE CORRESPONDING AUTHOR:}

Andleeb Ahangar,

Resident,

Department of Ophthalmology,

Government Medical College,

Srinagar.

E-mail: andleebali@gmail.com

Date of Submission: 28/03/2015.

Date of Peer Review: 30/03/2015.

Date of Acceptance: 28/05/2015.

Date of Publishing: 03/06/2015. 